\title{
The effect of heterogeneous dynamics of online users on information filtering
}

\author{
Bo-Lun Chen ${ }^{\mathrm{a}, \mathrm{b}, \mathrm{c}}$, An Zeng ${ }^{\mathrm{d}, *}$, Ling Chen ${ }^{\mathrm{a}, \mathrm{b}}$ \\ a Department of Computer Science, Yangzhou University of China, Yangzhou 225127, China \\ b Department of Computer Science, Nanjing University of Aeronautics and Astronautics of China, Nanjing 210016, China

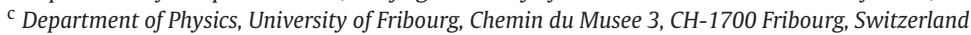 \\ d School of Systems Science, Beijing Normal University, Beijing 100875, China
}

The rapid expansion of the Internet requires effective information filtering techniques to extract the most essential and relevant information for online users. Many recommendation algorithms have been proposed to predict the future items that a given user might be interested in. However, there is an important issue that has always been ignored so far in related works, namely the heterogeneous dynamics of online users. The interest of active users changes more often than that of less active users, which asks for different update frequency of their recommendation lists. In this paper, we develop a framework to study the effect of heterogeneous dynamics of users on the recommendation performance. We find that the personalized application of recommendation algorithms results in remarkable improvement in the recommendation accuracy and diversity. Our findings may help online retailers make better use of the existing recommendation methods.

\section{Introduction}

With the fast development of the World Wide Web, our daily lives depend more and more on the Internet. However, how to find the information we need is not a simple problem [1]. The huge amount of online items such as the movies, books, bookmarks make it impossible for everyone to go over every item and find their favorite. Many approaches such as the collaborative filtering [2,3], matrix factorization [4-6], resource diffusion [7-9] have been intensively investigated recently. This so-called information filtering problem attracts researchers from computer science [10,11], physics [12-14], psychology [15,16], management [17] and so on. The research issues range from the recommendation accuracy [7] and diversity [9] to the sustainability of the whole system in evolution [18]. In this context, many recommendation algorithms have been proposed to help online users filter out irrelevant information and narrow down the search space $[19,20]$.

In the literature, the studies on recommender systems overwhelmingly focus on the recommendation techniques while the effect of online users' features on the recommendation process has received far less attention. Research on human dynamics has shown clearly that the behavior of online users is hetero-

\footnotetext{
* Corresponding author.

E-mail address: anzeng@bnu.edu.cn (A. Zeng).
}

geneous [21]. At individual level, the inter-event time of item selections exhibits the burst property, i.e. in some period users select items frequently while in some other period the time between two selections of a user can be very long [22]. At system level, the distribution of users activity is very broad, indicating that some users are very active while many other users are much less active $[23,24]$. Moreover, it has been revealed that online users are driven by different network growth mechanism when they establish new links in the network [25]. In this paper, we focus on how the heterogeneous dynamics of online users affects the information filtering process.

To evaluate the accuracy and diversity of recommendation, usually the real data (i.e. links) are randomly divided into two sets: the training set represents the known historical information that can be used by the recommendation algorithm; the probe set represents the unknown future information that is used to check the quality of the recommendation [1]. This implies that the number of links that the recommendation algorithm tries to predict for each user is proportion the cumulative degree of the user. However, this assumption could be problematic from practical point of view. Since the behavior of online users is very heterogeneous, some users could be very active and requires the recommendation list to be updated very often. Some less active users, on the other hand, may require less frequent update of the recommendation list [26]. Therefore, it is necessary to take into account the heteroge- 
neous dynamics of online users in data division and examine the performance of recommendation algorithms when the amounts of links for prediction are unequal for different users.

In this paper, we propose a heterogeneous data division model for the recommendation process. Instead of randomly dividing the links into the training set and probe set, the number of links for each user in the probe set is determined by the user's degree. The bias towards different degree is controlled by a tunable parameter. By implementing some representative recommendation algorithms, we find that different data division indeed significantly influences the evaluation results of the existing recommendation algorithms. Interestingly, if the number of probe links for large degree users is smaller than that from the random division (i.e. update the recommendation list more frequently for large degree users), the overall recommendation accuracy and diversity are improved.

\section{Related work}

In the literature, many researchers have considered the heterogeneity of online users when designing recommendation algorithms. Motivated by the observed significant difference between users' structural properties in the network, Zhang et al. proposed to remove redundant links for each user to extract the so-called information backbone [19]. Guan et al. observed that large degree users tend to select niche objects while small degree users tend to select popular objects. They thus proposed a personalized hybrid algorithm in which each user is assigned with a parameter to adjust the popularity of the objects shown in his/her personal recommendation list [27]. Zeng et al. argued that due to users' heterogeneity, they are carrying different amount of information for the recommendation algorithms. Accordingly, Zeng et al. identified some core users in the network and achieve $90 \%$ of the accuracy by taking only $20 \%$ of the core users' data into account [20]. Similar ideas have been extended to the study of online search engine. Sugiyama et al. proposed a personalized web search engine according to each user's need for relevant information without any user effort [28]. User heterogeneity will result in different information needs for each user's query. Therefore, the search results should be adapted to users with different information needs.

Compared to the recommendation algorithm design, user heterogeneity has less impact on the research on the data division. Most of the recommendation algorithms were validated based on the random data division, which obviously neglects the user heterogeneity in selecting items. In a recent review [1], it is mentioned that recommendation should be done with the data divided into the training set and probe set based on the time stamps on links. Focusing on the over-fitting problems for recommendation algorithms, Zeng et al. proposed a triple data division model in which the real data is divided into a training set, a learning set and a probe set [13]. The basic idea is to estimate users' parameters with the learning set and then applied the learned parameters to actually predict users' future objects in the probe set. Since the purchase behaviors which happened long time ago could not truly reflect the current interests of the target user, Guo et al. investigated the impact of the time window on the training set on the recommender algorithms [29]. In order to improve the diversity and accuracy of the recommender system, Song et al presented an improved hybrid information filtering of adopting the partial recent information in terms of the face that the recent behaviors are more effective to capture the users' potential interests, they also generated a series of training sets, each of which is treated as known information to predict the future links proven by the probe set [23].

Taken together, the research on users' heterogeneity mainly focuses on new algorithm design and the modification on the training set. One crucial direction has not been investigated so far, that is the effect of users' heterogeneity on the probe set. In this paper, we take into account the heterogeneous dynamics of online users and associate it with the length of the probe set (i.e. the number of items they will connect to in the future). We argue that active users and inactive users should have different amount of links in the probe set. This assumption not only helps us to understand better the recommendation process in real system but also provides us with a better implementation of the recommendation algorithms (i.e. update users' recommendation lists with different frequency).

\section{Data and model}

In this paper, we use two standard data sets which have been widely used to examine the performance of recommendation algorithms [29-31]. The first one is the Movielens data with 1682 movies (items) and 943 users (http://www.grouplens.org/). Users rate movies from 1 (worst) to 5 (best). Consistent with the literature, we consider the ratings higher than 2 as a link. Finally, 82520 links remain in the network. The second one is the Netflix data which is a random sample of the whole records of users ratings in Netflix.com (http://www.netflixprize.com/). It consists of 2294 users, 1891 movies, and 71074 links. Like Movielens, Netflix is also based on a 5-star rating system. With the same rating filtering process, we obtain 59464 links in Netflix data. Throughout this paper, we mainly present the results on Netflix data by figures and the results of both data sets are reported by tables.

In order to model the prediction process of the recommender systems, the above data (i.e. links) are divided into two parts: the training set $E^{T}$ represents the known information while the probe set $E^{P}$ represents the unknown information for prediction. Considering the heterogeneous dynamics of users, the division of links into these two sets are not completely random. As active and inactive users spend different amount of time online, their recommendation needs to be updated with different frequency. Some users are very active, their recommendation lists should be updated often. For the less active users, the generated recommendation lists could be used for a relatively longer time. Accordingly, we propose a data division model in which the amount of data in the probe set for each user is tunable. In each step, we randomly pick up a user $i$ with the probability $p_{i}=k_{i}^{\theta} / \sum_{j} k_{j}^{\theta}$. Then one of his/her links is randomly moved to the probe set. The process is terminated when the total amount of links in the probe set reaches $10 \%$ of the links in the original network. Here, $\theta$ is a tunable parameter. When $\theta=1$, the data division process reduces to the traditional random data division. When $\theta<1$, the links connecting to small degree users are more likely to be moved to the probe set, and vice versa.

\section{Recommendation algorithms}

In this paper, we consider the hybrid recommendation algorithm which combines the Mass diffusion and Heat conduction methods [9]. The user-item bipartite network is characterized by an adjacency matrix $A$ where the element $a_{i \alpha}$ equals to 1 if user $i$ has collected object $\alpha$, and 0 otherwise. The number of users and items is denoted as $N$ and $M$, respectively. Consistent with the literature, we use Latin and Greek letters, respectively, for userand item-related indices. To generate the recommendation list for a specific user $i$, the Hybrid method starts by assigning each item selected by user $i$ one unit of resource. This resource assignment can be represented by a vector $f_{i}$. The resources of these selected items then diffuse in the bipartite network for two steps with the transition matrix $W$. Each component in this matrix can be computed as 
$w_{\alpha \beta}=\frac{1}{k_{\alpha}^{1-\lambda} k_{\beta}^{\lambda}} \sum_{i=1}^{n} \frac{a_{\alpha i} a_{\beta i}}{k_{i}}$

where $k_{\alpha}$ is the degree of item $\alpha$ and $k_{i}$ is the degree of user $i$. The Hybrid method reduces to the standard Heat conduction method when $\lambda=0$, and the standard Mass diffusion method when $\lambda=1$. The final recommendation score of each item can be computed as $\vec{f}_{i}=f W$. The recommendation list for user $i$ is obtained by sorting $\vec{f}_{i}$ in descending order.

\section{Evaluation}

An effective recommendation should be able to accurately find the items that users like. In order to measure the recommendation accuracy, we make use of ranking score (RS). Specifically, $R S$ measures whether the ordering of the items in the recommendation lists matches users' real preference. As discussed above, the recommender system will provide each user with a ranking list which contains all his uncollected items. For a target user $i$, we calculate the position for each of his links in the probe set. If one of his uncollected item $\alpha$ is ranked at the 3th place and the total number of his uncollected items is 100 , the ranking score $R S_{i \alpha}$ will be 0.03 . In a good recommendation, the items in the probe set should be ranked higher, so that $R S$ will be smaller. Therefore, the mean value of the $R S$ over all the user-item relations in the probe set can be used to evaluate the recommendation accuracy as

$R S=\frac{1}{\left|E^{P}\right|} \sum_{i \alpha \in E^{P}} R S_{i \alpha}$.

The smaller the value of $R S$, the higher the recommendation accuracy.

Usually, online web sites only present the top part of the recommendation list to users. Therefore, a more practical recommendation accuracy measurement called precision $(P)$ is considered. For each user $i$, the precision of recommendation is calculated as

$P_{i}(L)=\frac{d_{i}(L)}{L}$,

where $d_{i}(L)$ represents the number of user $i$ 's deleted links contained in the top- $L$ places in the recommendation list. For the whole system, the precision $P(L)$ can be obtained by averaging the individual precisions over all users with at least one link in the probe set. The higher the value of $P(L)$, the better the recommendations.

Predicting what a user likes from the list of the most popular items is generally easy in recommendation, while uncovering users' very personalized preference (i.e. uncovering the unpopular items in the probe set) is much more difficult and important. Therefore, diversity should be considered as another significant aspects for recommender systems besides accuracy. In this paper, we employ two kinds of diversity measurement: personalization $(D)$ and novelty $(I)$.

The personalization mainly considers how users' recommendation lists are different from each other. It is usually measured by Hamming distance. We denote $C_{i j}(L)$ as the number of common items in the top- $L$ place of the recommendation list of user $i$ and $j$, their hamming distance can be calculated as

$D_{i j}(L)=1-\frac{C_{i j}(L)}{L}$.

$D_{i j}(L)$ is between 0 and 1 , which are respectively corresponding to the cases where $i$ and $j$ have the same or an entirely different recommendation list. By averaging $D_{i j}(L)$ over all pairs of users, we obtain the mean hamming distance $D(L)$. The more the recommendation list differs from each other, the higher the $D(L)$ is.
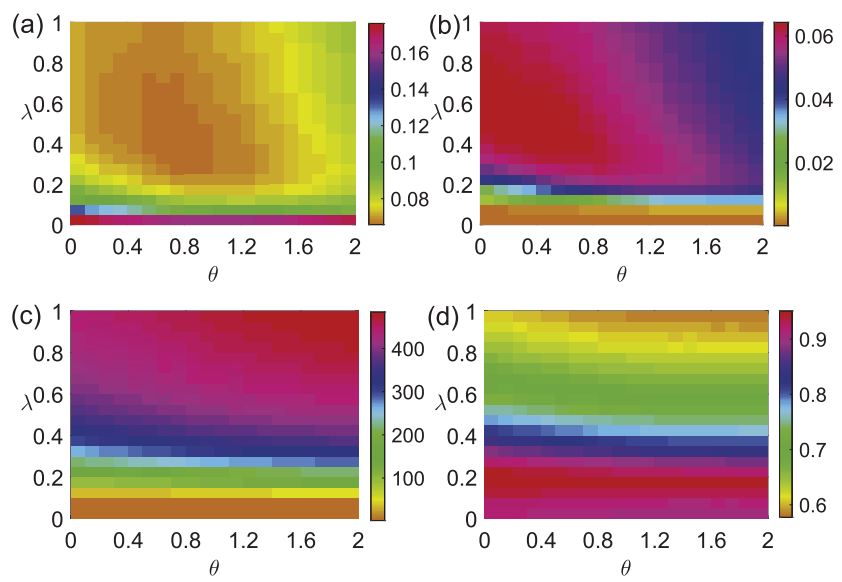

Fig. 1. (Color online.) (a) ranking score, (b) precision, (c) novelty and (d) personalization in $(\theta, \lambda)$ plane when the heterogeneous data division is used in the Netflix data. The recommendation algorithm is the Hybrid method. The results are averaged over 10 times of independent realizations.

The novelty measures the average degree of the items in the recommendation list. For those popular items, users may already get them from other channels. However, it is hard for the users to find the relevant but unpopular item. Therefore, a good recommender system should prefer to recommend small degree items. The metric novelty can be expressed as

$I_{i}(L)=\frac{1}{L} \sum_{\alpha \in O^{i}} k_{\alpha}$

where $O^{i}$ represents the recommendation list for user $i, k_{\alpha}$ represents the degree of the item $\alpha$. A low mean popularity $I(L)$ for the whole system indicates a high novel and unexpected recommendation of items.

\section{Results}

We start from investigating the dependence of the recommendation performance on parameter $\lambda$ and $\theta$ in Netflix. The results are shown as heatmaps in Fig. 1. In Ref. [9], it has been shown that the hybrid method of MD and $\mathrm{HC}$ algorithm can achieve a better $R S$ than the pure MD and pure $\mathrm{HC}$ methods when the real data is randomly divided into training set and probe set (i.e. $\theta=1$ ). In Fig. 1(a), we find that a minimum $R S$ still exists when $\theta \neq 1$ and the optimal RS under all possible value of $\lambda$ and $\theta$ happens in the region where $\theta<1$. Specifically, the optimal parameters are $\theta^{*}=0.7$ and $\lambda^{*}=0.4$ in Netflix and $\theta^{*}=0.1$ and $\lambda^{*}=0.5$ in Movielens. Compared to the random data division case, $R S$ is improved by $2.66 \%$ in Netflix and $13.64 \%$ in Movielens. These results indicate that introducing the heterogeneous data division mechanism can indeed enhance the general recommendation accuracy. As mentioned above, the heterogeneous data division is corresponding to personalized update of the recommendation list. In this sense, our results suggest that if personalized update of the recommendation list is implemented in practice the recommendation accuracy could be improved.

We then report the precision $P(L)$, novelty $I(L)$ and hamming distance $D(L)$ of the hybrid method in Fig. 1(b), (c), (d). All these metrics depend on the recommendation list $L$. In this paper, we set $L=10$ according to the literature [9]. The results of $P(L)$ in Fig. 1(b) confirm our finding that the heterogeneous data division could result in a higher recommendation accuracy. Compared with the precision in the random data division case, $P(L)$ in the heterogeneous data division is improved by $7.21 \%$ in Netflix and $9.23 \%$ in Movielens. The effect of $\theta$ on recommendation diversity, however, is minor, as shown in Fig. 1(c), (d). 

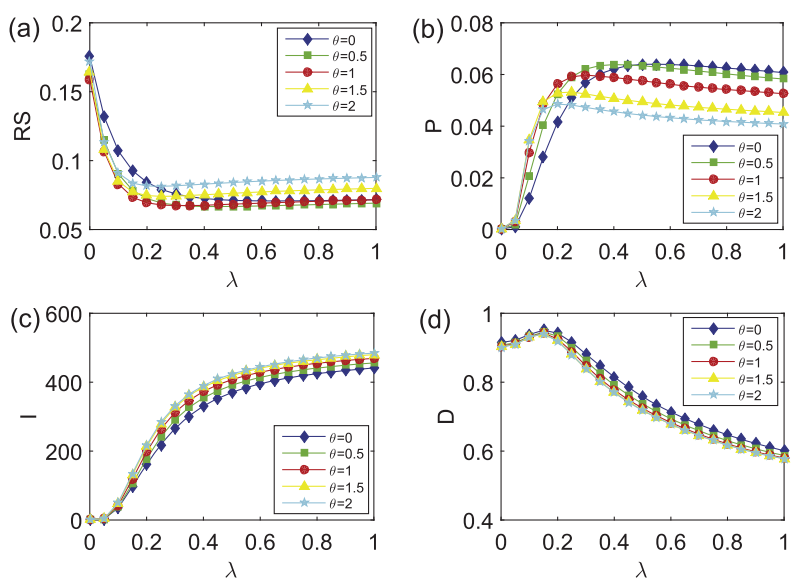

Fig. 2. (Color online.) The dependence of the (a) ranking score, (b) precision, (c) novelty and (d) personalization on $\lambda$ when different values of $\theta$ are set. The data in this figure is Netflix and the recommendation algorithm is the Hybrid method. The results are averaged over 10 times of independent realizations.
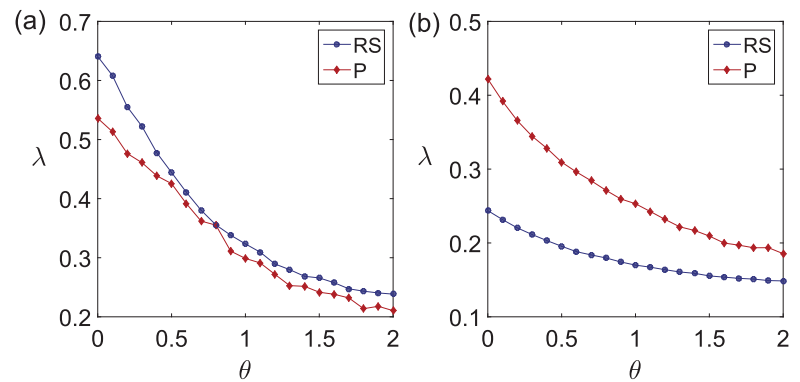

Fig. 3. (Color online.) The dependence of $\lambda^{*}$ (with respect to ranking score and precision) on $\theta$ in (a) Netflix and (b) Movielens. The results are averaged over 10 times of independent realizations.

In order to show the detailed effect of $\theta$ on the recommendation results, we select several $\theta$ values and plot the dependence of different recommendation metrics on $\lambda$ in Fig. 2 . We find that there is indeed an optimal $\lambda^{*}$ for ranking score and precision under each $\theta$. The value of $\lambda^{*}$ changes with $\theta$. The curves of different $\theta$ in novelty and personalization are highly overlapped. It confirms that there is only slight effect of $\theta$ on recommendation diversity.

The results in Fig. 2 indicate that $\lambda^{*}$ depends on $\theta$. We then quantitatively investigate this phenomenon in Fig. 3. We find that $\lambda^{*}$ decreases with $\theta$ in both Netflix and Movielens data sets. This is because the number of links in probe set for large degree users will increase when $\theta$ is large. As large degree users tend to select small degree items [21]. More links connecting to the small degree items will be placed in the probe set when $\theta$ is large. Therefore, the hybrid method needs to give more weight on the heat conduction method in order to predict these links more accurately. When ranking score and precision metrics are used to determine $\lambda^{*}$, its value can be a bit different. However, the decreasing trend of $\lambda^{*}$ is unchanged.

We further move to study the dependence of the optimal recommendation accuracy (when $\lambda^{*}$ is used) on $\theta$ in Fig. 4. We find that $R S^{*}$ reaches a best value when $\theta$ is smaller than 1 (note that $\theta=1$ is corresponding to the random data division). Similarly, $P^{*}$ also reaches an optimal value when $\theta<1$. These results suggest that when more links of small degree users are put in the probe set, the recommendation accuracy can be better. This phenomenon is natural. Table 1 shows the dependence of different recommendation metrics on the optimal $\lambda^{*}$ on several $\theta$ values. One can see that the results in Movielens are consistent with those in Netflix.
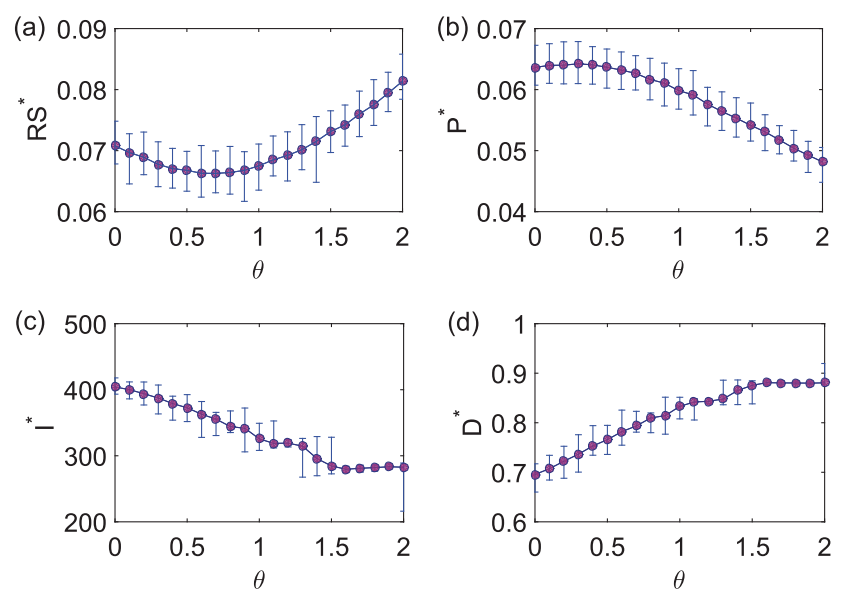

Fig. 4. (Color online.) The dependence of the optimal recommendation accuracy (when $\lambda^{*}$ is used) on $\theta$. The data in this figure is Netflix. The results are averaged over 10 times of independent realizations.

Table 1

Comparison of the $R S^{*}, P^{*}, D^{*}$ and $I^{*}$ (when $\lambda^{*}$ is used in the hybrid method) in the random data division $(\theta=1)$ and heterogeneous data division $(\theta \neq 1)$.

\begin{tabular}{llllll}
\hline \multirow{2}{*}{ Netflix } & & $\theta=0.5$ & $\theta=1.0$ & $\theta=1.5$ & $\theta=\theta_{\text {opt }}$ \\
& $R S^{*}$ & 0.0665 & 0.0676 & 0.0729 & $\mathbf{0 . 0 6 5 8}$ \\
& $P^{*}$ & 0.0635 & 0.0596 & 0.0542 & $\mathbf{0 . 0 6 3 9}$ \\
& $I^{*}$ & 374.7 & 325.2 & 285.9 & $\mathbf{2 7 9 . 2}$ \\
& $D^{*}$ & 0.763 & 0.834 & 0.874 & $\mathbf{0 . 8 8 1}$ \\
& & & & & \\
& $R S^{*}$ & 0.0728 & 0.0792 & 0.0851 & $\mathbf{0 . 0 6 8 4}$ \\
& $P^{*}$ & 0.1711 & 0.1592 & 0.1465 & $\mathbf{0 . 1 7 3 9}$ \\
& $I^{*}$ & 207.9 & 198.7 & 191.4 & $\mathbf{1 8 7 . 4}$ \\
& $D^{*}$ & 0.922 & 0.930 & 0.934 & $\mathbf{0 . 9 3 7}$ \\
\hline
\end{tabular}

In the literature, it has been pointed out that large degree items are more likely to be collected by small degree users [21]. When $\theta<1$, the number of links in the probe set for small degree users increases and small degree users tend to select large degree items. Therefore, the number of links connecting to the large degree items will increase in the probe set. As the links connecting to large degree items are easier to predict, the recommendation accuracy is improved. From the practical point of view, this result suggests us to update large degree users' recommendation list more frequently. In this way, their links for prediction in each round of recommendation will be reduced and the overall recommendation accuracy will be improved.

Finally, we study the effect of $\theta$ on the prediction accuracy of items with different degree. The ranking scores on the item degree under different $\theta$ are shown in Fig. 5. It can be seen that the ranking score decreases with item degree. This indicates that on average large degree items can be more accurately recommended than the small degree items. The insets also show the relation between ranking score and item degree, but with the $\mathrm{x}$-axis set as $\log$. There is a tendency that the ranking score of small degree items are influenced more by $\theta$. This indicates that the improved observed above are mainly due to the fact that the recommendation accuracy of the small degree items is improved.

In fact, the heterogeneous data division is corresponding to personalized update frequency of the recommendation list. Therefore, it can be regarded as a new method to implement the recommendation algorithm. We denote the hybrid method with the heterogeneous data division as $\mathrm{HH}$ method. We then compare its performance with the traditional recommendation algorithms including the Global Rank method (GR) [1], User-based Collaborative Filtering (UCF) [32] and Mass Diffusion (MD) [9]. In addition, two more recent algorithms are compared. The first one is called Most Popular Removal algorithm (MPR) which recommend items based 

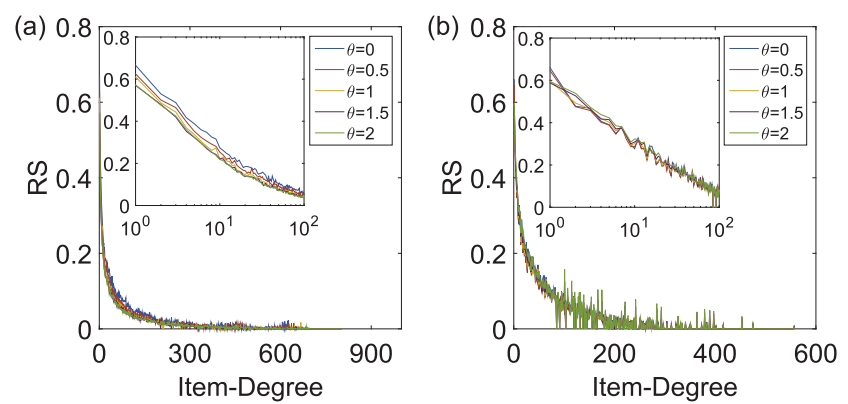

Fig. 5. (Color online.) The dependence of the ranking score on the item degree in (a) Netflix and (b) Movielens when different values of $\theta$ are set. Insets show ranking scores of items whose degree is no more than 100 on different $\theta$.

\section{Table 2}

Comparisons of average $R S^{*}, P^{*}, D^{*}$ and $I^{*}$ between the different algorithms. In the table, the optimal values for the data sets by the different algorithms are emphasized in bold-face.

\begin{tabular}{llllllll}
\hline \multirow{4}{*}{ Netflix } & & GR & UCF & MD & MPR & BHC & HH \\
& $R S^{*}$ & 0.0875 & 0.0783 & 0.0718 & 0.0721 & 0.0714 & $\mathbf{0 . 0 6 5 8}$ \\
& $P^{*}$ & 0.0415 & 0.0490 & 0.0530 & 0.0527 & 0.0581 & $\mathbf{0 . 0 6 3 9}$ \\
& $I^{*}$ & 508.78 & 484.51 & 468.34 & 471.71 & 326.18 & $\mathbf{2 7 9 . 2 4}$ \\
& $D^{*}$ & 0.3508 & 0.5193 & 0.5830 & 0.5663 & 0.8286 & $\mathbf{0 . 8 8 1 3}$ \\
& & & & & & & \\
Movielens & $R S^{*}$ & 0.1320 & 0.1024 & 0.1025 & 0.1027 & 0.0822 & $\mathbf{0 . 0 6 8 4}$ \\
& $P^{*}$ & 0.0852 & 0.1457 & 0.1375 & 0.1314 & 0.1628 & $\mathbf{0 . 1 7 3 9}$ \\
& $I^{*}$ & 355.40 & 312.98 & 314.32 & 286.54 & 199.63 & $\mathbf{1 8 7 . 4 0}$ \\
& $D^{*}$ & 0.5125 & 0.7642 & 0.7408 & 0.7688 & 0.9263 & $\mathbf{0 . 9 3 7 1}$ \\
\hline
\end{tabular}

on the information backbone of the user-item network [19]. The popularity of a link $i a$ is defined as $k_{u_{i}} k_{o_{a}}$, where $k_{u_{i}}\left(k_{o_{a}}\right)$ is the degree of user $u_{i}$ (item $o_{a}$ ). The method iteratively removes the most popular links to obtain the information backbone. In MPR, the recommendation is finally done with the MD algorithm after the network structure reduction. The second one is called Biased Heat Conduction method (BHC) which could greatly improves the accuracy of the standard Heat Conduction algorithm by considering the degree effects in the last step of the local heat conduction process [33]. The results of the recommendation metrics of these methods are shown in Table 2.

Accuracy is always the first consideration in evaluating a recommendation algorithm's performance. Comparing the result from the six methods, we can see that $\mathrm{HH}$ outperforms the other five algorithms in ranking score and precision. For example, compared with BHC, the $R S$ can be reduced by $7.84 \%$ for Netflix, $16.79 \%$ for Movielens. Compared with UCF, RS can be reduced by $15.96 \%$ for Netflix, 33.20\% for Movielens. The improvement is even larger if compared with GR, i.e. $24.80 \%$ for Netflix, $48.18 \%$ for Movielens. In this sense, HH can provide the best accuracy.

Besides accuracy, novelty and diversity are two other important metrics. As shown in Table 2, HH outperforms GR in $D(L)$ by $151.23 \%$ for Netflix, $82.85 \%$ for Movielens. When comparing HH with UCF and BHC, $D(L)$ can be improved by $69.71 \%$ and $6.36 \%$ respectively for Netflix, and $22.62 \%$ and $1.17 \%$ respectively for Movielens. Taken together, $\mathrm{HH}$ achieves the best recommendation performance among all the considered methods.

\section{Conclusion}

In this paper, we propose a new framework to evaluate the performance of recommendation algorithms. Compared with the traditional method, the way of real links divided into the training set and probe set is different. The amount of links for each user to be placed in the probe set is based on his/her degree with a tunable parameter which controls whether small degree users or large degree users tend to have more links in the "future". We consider several representative recommendation algorithms and find that the new evaluation framework significantly influences the estimated accuracy and diversity of the recommendation methods. Moreover, if the number of probe set links for large degree users is smaller than that from the random division, the overall recommendation accuracy and diversity are improved. Our new framework can be interpreted by the update frequency of the recommendation lists in practice. Our results show that the recommendation lists of the large degree users need to be updated more frequently to achieve higher user satisfactory. In this sense, our findings are meaningful in application as it helps online retailers make better use of the existing recommendation methods.

Our results indicate that the performance of the existing recommendation methods are only tested in a very special case in which each link of the large degree users and small degree users is equally likely to be put in the probe set. Therefore, reexamining the performance of all the existing methods under the new framework in this paper would be an interesting extension. Moreover, a more general data division framework could make the probability of a link to be put in the probe set depends on both user degree and item degree. Such framework could be used to investigate also how often items of different popularity should be included in the recommendation list. This problem asks for future research.

\section{Acknowledgements}

This research was supported in part by the National Natural Science Foundation of China under grant Nos. 61379066, 61379064, 61472344, 61402395, Natural Science Foundation of Jiangsu Province under contracts BK20130452, BK20140492, and China Scholarship Council. A.Z. acknowledges the support from the Youth Scholars Program of Beijing Normal University (grant No. 2014NT38).

\section{References}

[1] L. Lu, M. Medo, C.H. Yeung, Y.-C. Zhang, Z.-K. Zhang, T. Zhou, Phys. Rep. 519 (2012) 1.

[2] J.L. Herlocker, J.A. Konstan, K. Terveen, J.T. Riedl, ACM Trans. Inf. Syst. Secur. 22 (2004) 5.

[3] J.B. Schafer, D. Frankowski, J. Herlocker, S. Sen, The adaptive web, in: Collaborative Filtering Recommender Systems, Springer, 2007, p. 291.

[4] Y. Koren, R. Bell, C. Volinsky, Computer 8 (2009) 30.

[5] H. Ma, H.-X. Yang, U. L, R. Michael, I. King, in: Proc. ACM Conference on Information and Knowledge Management, ACM, 2008, p. 931.

[6] M. Jamali, M. Ester, in: Proc. ACM Conference on Information and Knowledge Management, ACM, 2010, p. 135.

[7] T. Zhou, J. Ren, M. Medo, Y.-C. Zhang, Phys. Rev, E 76 (2007) 046115.

[8] Y.-C. Zhang, M. Blattner, Y.-K. Yu, Phys. Rev. Lett. 99 (2007) 154301.

[9] T. Zhou, Z. Kuscsik, J.-G. Liu, M. Medo, J.R. Wakeling, Y.-C. Zhang, Proc. Natl. Acad. Sci. 107 (2010) 4511.

[10] B. Sarwar, G. Karypis, J. Konstan, J. Riedl, in: Proc. ACM Conference on Information and Knowledge Management, ACM, 2001, p. 285.

[11] F. Ricci, L. Rokach, B. Shapira, in: Recommender Systems Handbook, Springer, 2011, p. 1.

[12] J. Ren, T. Zhou, Y.-C. Zhang, Europhys. Lett. 82 (5) (2008) 58007.

[13] A. Zeng, A. Vidmer, M. Medo, Y.-C. Zhang, Europhys. Lett. 105 (5) (2014) 58002.

[14] W. Zeng, A. Zeng, M.-S. Shang, Y.-C. Zhang, PLoS ONE 8 (11) (2013) e79354.

[15] D. Reitter, C. Lebiere, in: Proceedings of the Twenty-Sixth AAAI Conference on Artificial Intelligence, AAAI, 2012, p. 242.

[16] J. Zhou, J. Yin, T. Chen, X.-W. Ding, Z.-F. Gao, M.-W. Shen, PLoS ONE 6 (9) (2011) e23873.

[17] J. Yang, J. Kim, W. Kim, Y.H. Kim, PLoS ONE 7 (11) (2012) e49126.

[18] A. Zeng, C.H. Yeung, M.-S. Shang, Y.-C. Zhang, Europhys. Lett. 97 (2012) 18005.

[19] O.-M. Zhang, A. Zeng, M.-S. Shang, PLoS ONE 8 (5) (2013) e62624.

[20] W. Zeng, A. Zeng, H. Liu, M.-S. Shang, T. Zhou, Sci. Rep. 4 (2014) 6140.

[21] M.-S. Shang, L. Lu, Y.-C. Zhang, T. Zhou, Europhys. Lett. 90 (4) (2010) 48006.

[22] P.G. Campos, F. Díez, A. Bellogín, in: Proc. ACM Conference on Information and Knowledge Management, ACM, 2011, p. 29.

[23] W.-J. Song, Q. Guo, J.-G. Liu, Physica A 416 (2014) 192.

[24] Y.-B. Zhou, A. Zeng, W.-H. Wang, PLoS ONE 10 (3) (2015) e0120735.

[25] L. Xiang, Q. Yuan, S.-W. Zhao, L. Chen, X.-T. Zhang, Q. Yang, J. Sun, in: Proc. ACM Conference on Information and Knowledge Management, ACM, 2010, p. 723.

[26] G. Bianconi, P. Laureti, Y.-K. Yu, Y.-C. Zhang, Physica A 332 (2004) 519. 
